\title{
Influence of process parameters on the deep drawing of stainless steel
}

\author{
R. Padmanabhan ${ }^{\mathrm{a}, *}$, M.C. Oliveira ${ }^{\mathrm{a}}$, J.L. Alves ${ }^{\mathrm{b}}$, L.F. Menezes ${ }^{\mathrm{a}}$ \\ ${ }^{a}$ CEMUC, Department of Mechanical Engineering, University of Coimbra, Polo II, Pinhal de Marrocos, 3030-201 Coimbra, Portugal \\ ${ }^{\mathrm{b}}$ Department of Mechanical Engineering, University of Minho, Campus de Azurém, 4800-058 Guimarães, Portugal
}

Received 19 September 2006; received in revised form 13 June 2007; accepted 14 June 2007

Available online 23 July 2007

\begin{abstract}
Optimization of process parameters in sheet metal forming is an important task to reduce manufacturing cost. To determine the optimum values of the process parameters, it is essential to find their influence on the deformation behaviour of the sheet metal. The significance of three important process parameters namely, die radius, blank holder force and friction coefficient on the deep-drawing characteristics of a stainless steel axi-symmetric cup was determined. Finite element method combined with Taguchi technique form a refined predictive tool to determine the influence of forming process parameters. The Taguchi method was employed to identify the relative influence of each process parameter considered in this study. A reduced set of finite element simulations were carried out as per the Taguchi orthogonal array. Based on the predicted thickness distribution of the deep drawn circular cup and analysis of variance test, it is evident that die radius has the greatest influence on the deep drawing of stainless steel blank sheet followed by the blank holder force and the friction coefficient. Further, it is shown that a blank holder force application and local lubrication scheme improved the quality of the formed part.
\end{abstract}

(C) 2007 Elsevier B.V. All rights reserved.

Keywords: Deep drawing; Process parameters; Stainless steel; FEM; Taguchi technique; ANOVA

\section{Introduction}

Sheet metal forming is a technique by which most body parts are produced in automobile industries. In sheet metal forming, a thin blank sheet is subjected to plastic deformation using forming tools to conform to a designed shape. During this process, the blank sheet is likely to develop defects if the process parameters are not selected properly. Therefore, it is important to optimize the process parameters to avoid defects in the parts and to minimize production cost. Optimization of the process parameters such as die radius, blank holder force, friction coefficient, etc., can be accomplished based on their degree of importance on the sheet metal forming characteristics. In this investigation, a statistical approach based on Taguchi technique was adopted to determine the degree of importance of each of the process parameters on the thickness distribution of deep drawn circular cup. Taguchi method [1] has been applied in forming studies to design the experiments and determine the influence of process parameters on characteristics of the formed part [2-8].

\footnotetext{
* Corresponding author. Tel.: +351 239790715; fax: +351 239790701.

E-mail address: padmanabhan@dem.uc.pt (R. Padmanabhan).
}

\section{Taguchi technique}

Taguchi proposed several approaches to experimental designs called Taguchi method [1]. This method utilizes an orthogonal array, which is a form of fractional factorial design containing a representative set of all possible combination of experimental conditions. Using Taguchi method, a balanced comparison of levels of the process parameters and significant reduction in the total number of required simulations can both be achieved. Mark Colgan and John Monaghan reported preliminary study made on the influence of process parameters in deep-drawing process [8]. Taguchi L8 orthogonal array was used to investigate the effect of seven process parameters in eight experiments. The process parameters studied were, the punch radius, the die radius, the blank holder force, the lubricant type, the lubrication position, the punch velocity and the draw depth. Two levels (low, high) were used for each parameter. The results indicate that the punch/die radii have the greatest effect on the thickness of the deformed mild steel cups compared to other process parameters.

In the present study, Taguchi method of experimental design was used to plan the numerical simulations. In Taguchi design, 
Table 1

Process parameters and their levels

\begin{tabular}{llcr}
\hline Process parameter & \multicolumn{2}{c}{ Level } & \\
\cline { 2 - 4 } & 1 & 2 & 3 \\
\hline Die radius $(R)(\mathrm{mm})$ & 14 & 18 & 22 \\
BHF $(\mathrm{kN})$ & 85 & 95 & 105 \\
Friction coefficient $(\mu)$ & 0.08 & 0.14 & 0.2 \\
\hline
\end{tabular}

Table 2

Orthogonal array (L9) of Taguchi method

\begin{tabular}{llll}
\hline Experiment & \multicolumn{2}{l}{ Parameter } & $\mu$ \\
\cline { 2 - 4 } & $R$ & BHF & 1 \\
\hline 1 & 1 & 1 & 2 \\
2 & 1 & 2 & 3 \\
3 & 1 & 3 & 2 \\
4 & 2 & 1 & 3 \\
5 & 2 & 2 & 1 \\
6 & 2 & 3 & 3 \\
7 & 3 & 1 & 1 \\
8 & 3 & 2 & 2 \\
9 & 3 & 3 & \\
\hline
\end{tabular}

using two levels of each factors form screening experiments to determine a model of the system to a linear approximation. By this, the least influential parameters are identified and eliminated before the most influential process parameters can be further studied. To capture non-linear effects more than two levels [2-7] are required to predict the factor's behaviour since two levels $[7,8]$ produce only linear effects. Hence, three levels of the process parameters were used in this study to capture the non-linear effects in the experimental design.

There are many factors, both process and material parameters, which influence deep-drawing process. In this study, deep drawing of LPG bottle was considered as a case and hence the choice of a high corrosion resistant and heat resistant material such as AISI 304 stainless steel. Among all process parameters, blank holder force, die radius and friction coefficient play an important role in the quality of the formed component and hence the above parameters are considered in this study. To evaluate, three levels are chosen for each parameter. The levels are based on the process window and conform to low, medium and high feasible values. Other process parameters such as punch radius, draw depth, etc. were fixed to the recommended values in the simulations. Table 1 shows the chosen process parameters and their levels used in the FE simulations.

The high order interactions among the above three factors are assumed negligible and the information on the main effects can be obtained by running $3^{3}=27$ experiments. However, the appropriate Taguchi orthogonal array for the above three parameters with three levels is L9 to conduct nine simulations, Table 2. The first column represents the number of simulation and the subsequent columns represent the process parameters and the rows represent simulations with the levels of each parameter.
After the experiments are designed with various combinations of process parameter levels, FE simulations were carried out to predict the deformation behaviour of the blank sheet. The results obtained from the FE simulations were treated using statistical approach namely, ANOVA method. The purpose of using ANOVA is to elucidate the parameters that govern the deep-drawing process that markedly influence the thickness distribution. This will yield information about the impact of each parameter on the results predicted by the finite element method. Consequently, the degree of importance of each process parameter in the deformation behaviour of the blank sheet can be determined.

\section{Deep-drawing simulations of stainless steel cup}

The formability of a blank sheet depends on the process parameters such as blank holder force, lubrication, punch and die radii, die-punch clearance, in addition to mechanical properties and thickness of the sheet metal and part's geometry. Fracture and wrinkle are the two major modes of failure in sheet metal parts. Hence, using proper blank holder force is an essential criterion to restrict wrinkling tendency and avoid tearing of the blank sheet. Previous research on these failure modes highlights the role of blank holder force in forming and suggests different blank holder force application schemes to eliminate these failure modes [9-14]. However, a constant blank holder force is used in this study for simplicity. In addition to the blank holder force, the die radius and the friction coefficient control the metal flow into the die cavity in deep-drawing process [15-17]. The flow of material into the die cavity reduces with small die radius while a large die radius lead to wastage in trimming excess material and induces springback characteristic. An appropriate die radius allows smooth flow of materials on one hand and reduces springback and material wastage. Similarly, proper lubrication condition enhances the flow of material into the die cavity. The quality of the formed part is dictated by the degree of influence of these process parameters used in sheet metal forming process. One of the quality criterions in sheet metal formed parts is thickness distribution. Failure in deep drawn partusually occurs by thinning, therefore, it is important to determine the variation of strain in thickness direction during deformation $[18,19]$. The objective is to reduce thickness variation in the deep drawn part, i.e. to maximize the minimum thickness and minimize the maximum thickness. Thus, in the present investigation, an attempt has been made to study the effect of these three important process parameters, namely blank holder force, die radius and friction coefficient, on the thickness variation of the part.

Numerical studies are capable of revealing the deformation pattern of the blank sheet, characterizing materials under industrial deep-drawing conditions, to result in an optimal final solution. A range of forming parameters can be used in the finite element simulations and the optimal values can be predicted at low CPU cost [20]. Preliminary simulations were carried out to determine the range of each process parameter. This will allow identifying the levels for the parameters as the level need to be in an operational range of the deep-drawing process. 


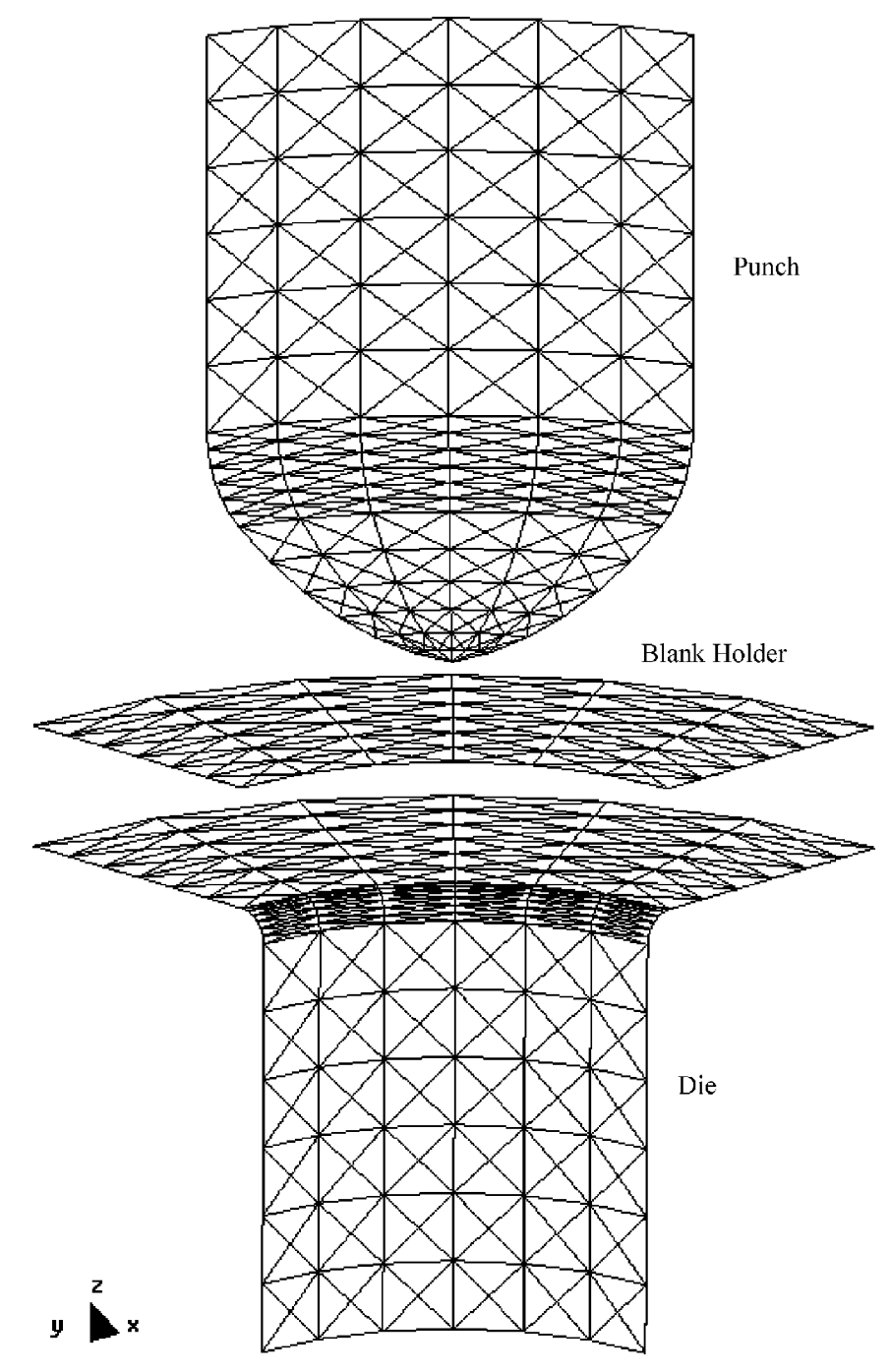

Fig. 1. Tools used in the simulations.

Deep-drawing simulations were carried out using the in-house finite element code DD3IMP (abbreviation of deep-drawing 3D implicit code), which is specifically developed to simulate sheet metal forming processes [21]. A quarter of the model was employed in the simulations due to symmetry of problem. The coefficient of Coulomb friction between the deforming blank sheet and forming tools (die, punch and blank holder) was assumed to be same and maintained at three levels (Table 1). The forming tools, defined by parametric Bézier surfaces, are considered to behave rigidly. For clarity in visualizing, the forming tools used in the simulation are depicted using triangular elements in the post-processor, Fig. 1. Fig. 2 illustrates the geometry of the tools used in circular cup deep-drawing simulations.

Stainless steel has excellent corrosion resistance and mechanical properties, the properties most essential for applications such as LPG bottles. Despite its high strength capabilities, it retains good formability characteristics compared to other high strength materials. An austenitic grade AISI 304 stainless steel is used for this study [22]. The work-hardening behaviour is considered isotropic and described by the Swift

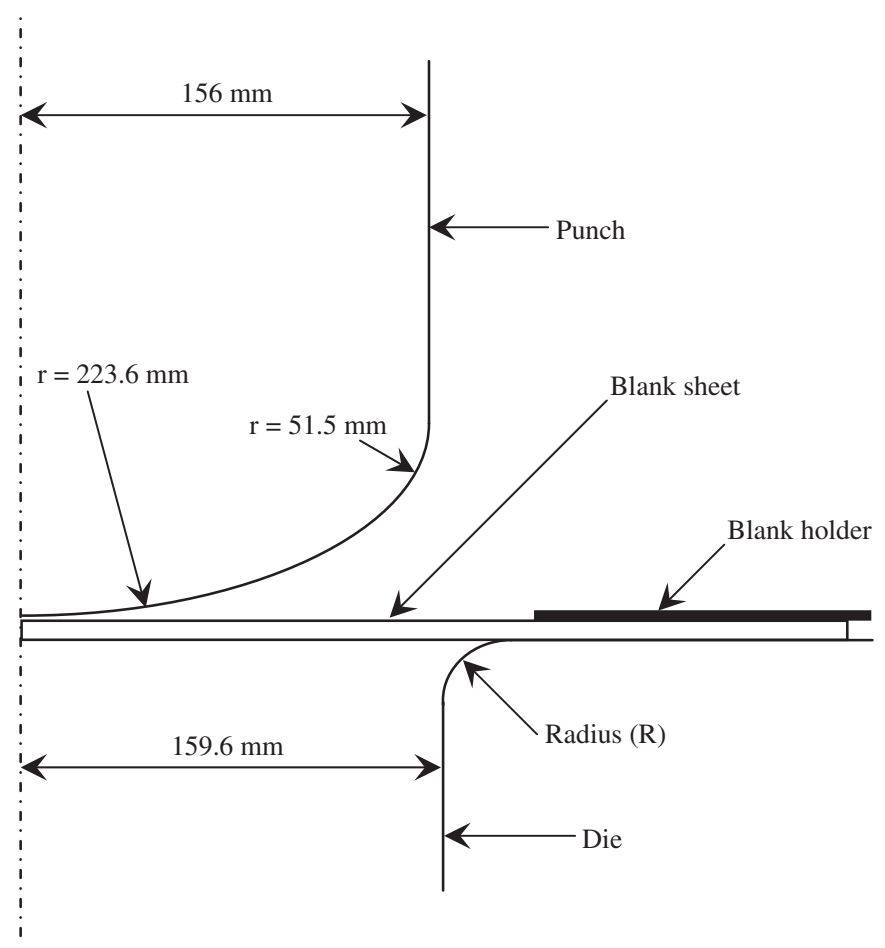

Fig. 2. Geometry of the forming tools.

power law: $\bar{\sigma}=K\left(\varepsilon_{0}+\bar{\varepsilon}^{P}\right)^{n}$, with the plastic anisotropy described by the Hill48's quadratic yield criterion [23]. The elastic properties are: Young's modulus $E=200 \mathrm{GPa}$, and Poisson ratio $v=0.33$. The hardening parameters are: strength coefficient $K=1330 \mathrm{MPa}$ and the strain-hardening exponent $n=0.35$, and $\varepsilon_{0}=0.009$. The initial blank sheet has a radius of $320 \mathrm{~mm}$ and a thickness of $3.5 \mathrm{~mm}$. The blank is meshed with eight node solidfinite elements. An in-plane average FE mesh size of $7 \mathrm{~mm}$ was used with two layers through thickness.

\section{Results and discussion}

\subsection{Punch force evolution}

Fig. 3 shows the evolution of punch force on the deep drawing of the axi-symmetric cup. Marginal differences in the punch forces are observed between the experiments. In the first three experiments, when the die radius was at lowest level, higher punch force evolution occurred compared to the remaining six cases. The punch force drops rapidly after about $190 \mathrm{~mm}$ of punch displacement indicating the onset of plastic instability in the part. In experiment 3 (most unfavourable combination of chosen process parameters), the cup fails at the punch radius at about $240 \mathrm{~mm}$ which is evident from the distinct drop in the punch force. Whereas in other cases (experiments 4-9), the punch force continue to increase until the end of deep-drawing process $(270 \mathrm{~mm})$. It is apparent that the die radius has a very important role in deep-drawing process. The friction coefficient and blank holder force have moderate influence on the deepdrawing process. 


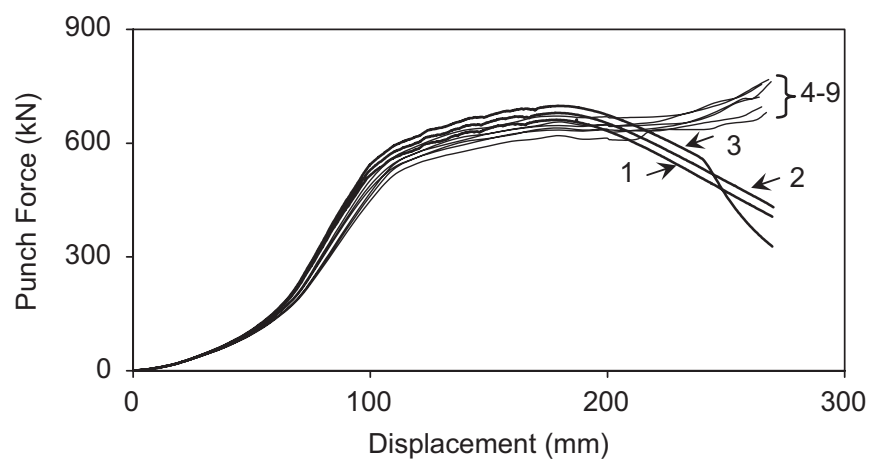

Fig. 3. Punch force evolution.

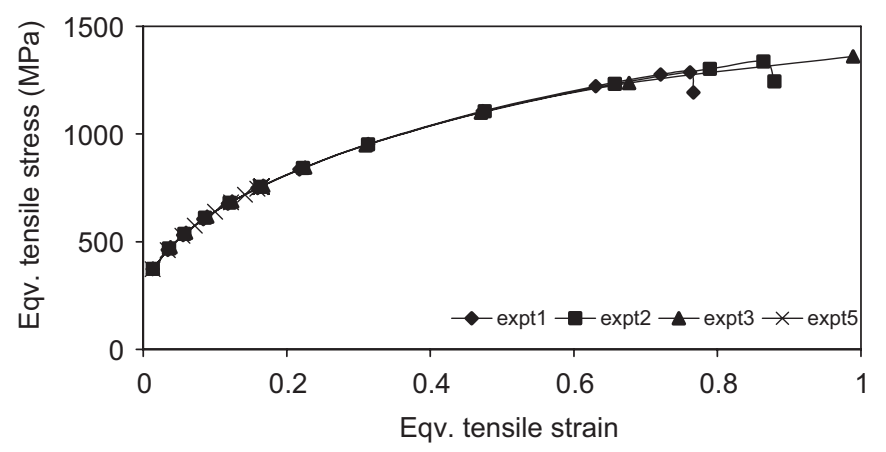

Fig. 4. Equivalent tensile stress-tensile strain curve for a node of interest.

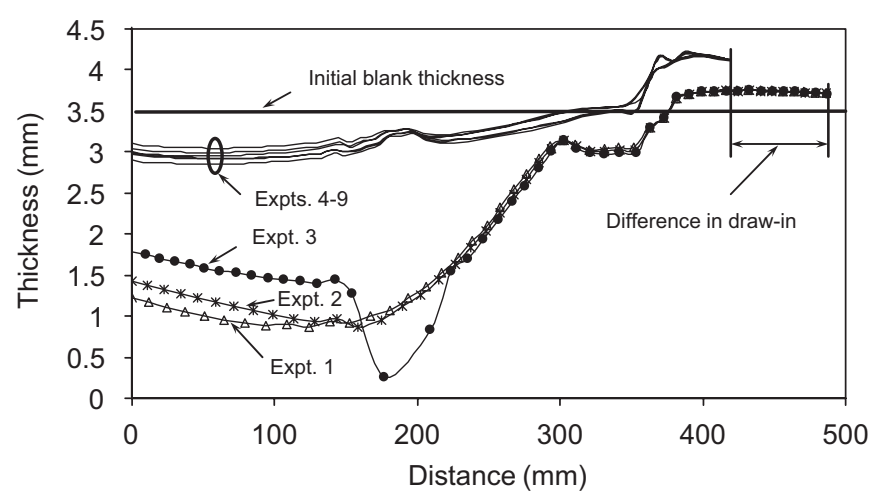

Fig. 5. Thickness variation in the circular cup.

Fig. 4 shows the equivalent tensile stress-tensile strain curves for a node of interest, located at the punch radius, in four different experiments. Experiments 1-3 utilized the lowest die radius and higher levels of other two factors. As a consequence, the blank sheet is subjected to very high equivalent tensile stresses indicating failure by tearing at the punch radius (Fig. 5). For comparison, case 5 that utilized a favourable combination of process parameters was chosen. The node of interest in this particular case is subjected to equivalent tensile stress and strain values well within the acceptable limit.

\subsection{Influence of process parameters on thickness distribution}

The quality characteristics chosen for the experiment should reflect as accurately as possible the design parameters under study. Thickness is one of the major quality characteristic in sheet metal formed part, especially for the studied geometry which is analogous to LPG bottle forming. The thickness is unevenly distributed in the part after deep drawing. Generally, the thickness is uniform at the bottom face of the punch, minimum at the punch radius and vertical surface, and thicker at the flange area.

Existence of thickness variation from the production stage may cause stress concentration in the part leading to acceleration of damage mechanism. Optimal values of process parameters and their combination results in high quality parts. Fig. 5 shows the thickness variation in the deep drawn part along OX direction. Wall thinning occurs at the bottom of the cup and thickening occurs near the top and at the flange. Near the top of the cup section and at the flange, blank thickening occurs due to the friction at die-blank sheet interface and the circumferential forces. The thickness variation is limited in experiments 4-9 where a favourable combination of process parameters and their levels were used. The maximum thinning was observed to be less than $16 \%$ of the initial thickness. Whereas the thickness variation is unacceptable in experiments $1-3$. Thinning is high in these set of experiments due to unfavourable combination of levels of process parameters. The material flow into the die cavity is restricted and hence the difference in the draw-in, as indicated in Fig. 5.

\subsection{Anova}

Calculations of result averages and averages for factor level effects, which only involve simple arithmetic operations produce answers to major questions that were unconfirmed in the preliminary stages of the investigation. However, questions concerning the influence of factors on the variation of thickness in terms of discrete proportion can only be obtained by performing analysis of variance (ANOVA). In this paper, Pareto ANOVA [24], which quantifies the importance of each process parameter in deep-drawing process, was used. The traditional method of calculating average factor effects and thereby determining the desirable factor levels is to look at the simple averages of the results. Although average calculations are relatively simple, it does not capture the variability of results with a trial condition. A better way to compare the sheet metal behaviour to deep drawing is to use the mean squared deviation, which combines effects of both average and standard deviation of the results. In order to increase the robustness of design against noises and to accommodate wide ranging data, Taguchi [1] recommended a logarithmic transformation of MSD (called the signal-to-noise ratio) for analysis of results. When the $S / N$ ratio is used for results analysis, the optimum condition identified from such analysis is more likely to produce consistent performance. The thickness of the deep drawn part was measured along three directions, $O X, O Y$, and $X Y$ and the average 
Table 3

ANOVA data table

\begin{tabular}{|c|c|c|c|c|c|}
\hline Parameter & Level & Expt no. & Thickness, $Y$ & $S / N$ & $S / N_{i j}$ \\
\hline \multicolumn{6}{|l|}{ Die radius } \\
\hline & & 1 & 2.762667 & 8.826571 & \\
\hline \multirow[t]{3}{*}{ Level 1} & $14 \mathrm{~mm}$ & 2 & 2.906288 & 9.266773 & 9.287384 \\
\hline & & 3 & 3.079218 & 9.768809 & \\
\hline & & 4 & 3.316861 & 10.41455 & \\
\hline \multirow[t]{3}{*}{ Level 2} & $18 \mathrm{~mm}$ & 5 & 3.2763 & 10.30767 & 10.35241 \\
\hline & & 6 & 3.286633 & 10.33502 & \\
\hline & & 7 & 3.370214 & 10.55315 & \\
\hline \multirow[t]{2}{*}{ Level 3} & $22 \mathrm{~mm}$ & 8 & 3.317709 & 10.41677 & 10.50367 \\
\hline & & 9 & 3.365541 & 10.5411 & \\
\hline \multicolumn{6}{|c|}{ Blank holder force } \\
\hline & & 1 & 2.762667 & 8.826571 & \\
\hline \multirow[t]{3}{*}{ Level 1} & $85 \mathrm{kN}$ & 4 & 3.316861 & 10.41455 & 9.931422 \\
\hline & & 7 & 3.370214 & 10.55315 & \\
\hline & & 2 & 2.906288 & 9.266773 & \\
\hline \multirow[t]{3}{*}{ Level 2} & $95 \mathrm{kN}$ & 5 & 3.2763 & 10.30767 & 9.997071 \\
\hline & & 8 & 3.317709 & 10.41677 & \\
\hline & & 3 & 3.079218 & 9.768809 & \\
\hline \multirow[t]{2}{*}{ Level 3} & $105 \mathrm{kN}$ & 6 & 3.286633 & 10.33502 & 10.21498 \\
\hline & & 9 & 3.365541 & 10.5411 & \\
\hline \multicolumn{6}{|c|}{ Friction coefficient } \\
\hline & & 1 & 2.762667 & 8.826571 & \\
\hline \multirow[t]{3}{*}{ Level 1} & 0.08 & 6 & 3.286633 & 10.33502 & 9.859454 \\
\hline & & 8 & 3.317709 & 10.41677 & \\
\hline & & 2 & 2.906288 & 9.266773 & \\
\hline \multirow[t]{3}{*}{ Level 2} & 0.14 & 4 & 3.316861 & 10.41455 & 10.07414 \\
\hline & & 9 & 3.365541 & 10.5411 & \\
\hline & & 3 & 3.079218 & 9.768809 & \\
\hline \multirow[t]{3}{*}{ Level 3} & 0.20 & 5 & 3.2763 & 10.30767 & 10.20988 \\
\hline & & 7 & 3.370214 & 10.55315 & \\
\hline & & & & \multicolumn{2}{|c|}{$\overline{S / N}=10.04782$} \\
\hline
\end{tabular}

values in each direction are presented in Table 3 against three experiments for eachlevel, respectively.

The $S / N$ ratio is used to measure the thickness deviation. The $S / N$ ratio is explained as: $-10 \log (\mathrm{MSD})$, where MSD is the mean square deviation $\left(1 / n \sum_{i=1}^{n}\left(1 / Y_{i}^{2}\right)\right)$ for the output characteristics; $n$ is the number of experiments and $Y$ is the measured value of thickness. The overall mean $S / N$ ratio is expressed as

$\overline{S / N}=\frac{1}{9} \sum_{i=1}^{9}(S / N)_{i}$.

The sum of squares due to variation about the overall mean is

$S S=\sum_{i=1}^{9}\left((S / N)_{i}-\overline{S / N}\right)^{2}$.

The calculated value for this expression is 0.98534 . For the $i$ th process parameter, the sum of squares due to variation about the mean is

$S S_{i}=\sum_{j=1}^{3}\left((S / N)_{i j}-\overline{S / N}\right)^{2}$.
Table 4

Contribution of process parameters on the deep-drawing process

\begin{tabular}{llc}
\hline Process parameter & Sum of squares $\left(S S_{i}\right)$ & \% Contribution \\
\hline Die radius & 0.878841 & 89.2 \\
Friction coefficient & 0.062437 & 6.3 \\
Blank holder force & 0.044065 & 4.5 \\
\hline
\end{tabular}

The calculated value for each process parameter is given in Table 4. The percentage contribution of individual process parameter on the deep-drawing process can be calculated by

$\%$ Contribution $_{i}=\frac{S S_{i}}{S S} \times 100$.

The degrees of importance of each process parameter considered, namely, die radius, blank holder force and friction coefficient is given in Table 4.

Die radius has greatest influence on the deep drawing of stainless steel blank sheet with $89.2 \%$ influence followed by the friction coefficient with $6.3 \%$ and the blank holder force with $4.5 \%$. Further optimization of the process parameters value can be made based on the degree of importance of the factors on the deep-drawing behaviour of the circular cup in order to obtain high quality parts. 


\section{Conclusions}

This paper illustrates the use of FEM with Taguchi technique to determine the proportion of contribution of three important process parameters in the deep-drawing process namely die radius, blank holder force and friction coefficient. FEM and Taguchi technique forms an effective tools combination to predict the influence of process parameters. The analysis of variance (ANOVA) was carried out to examine the influence of process parameters on the quality characteristics (thickness variation) of the circular cup and their percentage contribution were calculated. The die radius $(89.2 \%)$ has major influence on the deep-drawing process, followed by friction coefficient $(6.3 \%)$ and blank holder force $(4.5 \%)$. Further optimization of these process parameters value can be facilitated based on the degree of influence of the factors on the deep-drawing behaviour of the circular cup in order to improve the quality of the part. The study provided an insight into the deep drawing of stainless steel blank sheet. The quality of the drawn part depends on the forming conditions and the optimal value of process parameters and their favourable combination is important for deep-drawing process.

\section{Acknowledgement}

The authors are grateful to the Portuguese Foundation for Science and Technology (FCT) for the financial support for this work, through the Program POCI 2010.

\section{References}

[1] G. Taguchi, S. Konishi, Taguchi method, orthogonal arrays and linear graphs, Tools for Quality Engineering, American Supplier Institute, 1987 pp. $35-38$.

[2] X. Duan, T. Sheppard, Influence of forming parameters on the final subgrain size during hot rolling of aluminium alloys, J. Mater. Process. Technol. 130-131 (2002) 245-249.

[3] S.W. Lee, Study on the forming parameters of the metal bellows, J. Mater. Process. Technol. 130-131 (2002) 47-53.

[4] D.C. Ko, D.H. Kim, B.M. Kim, J.C. Choi, Methodology of preform design considering workability in metal forming by the artificial neural network and Taguchi method, J. Mater. Process. Technol. 80-81 (1998) 487-492.

[5] D.-C. Koa, D.-H. Kim, B.-M. Kim, Application of artificial neural network and Taguchi method to preform design in metal forming considering workability, Int. J. Mach. Tools Manufacture 39 (1999) $771-785$.
[6] K. Park, Y. Kim, The effect of material and process variables on the stamping formability of sheet materials, J. Mater. Process. Technol. 51 (1995) 64-78.

[7] R.S. Chen, H.H. Lee, C.Y. Yu, Application of Taguchi's method on the optimal process design of an injection molded PC/PBT automobile bumper, Compos. Struct. 39 (3-4) (1997) 209-214.

[8] M. Colgan, J. Monaghan, Deep drawing process: analysis and experiment, J. Mater. Process. Technol. 132 (2003) 35-41.

[9] E.J. Obermeyer, S.A. Majlessi, A review of recent advances in the application of blank-holder force towards improving the forming limits of sheet metal parts, J. Mater. Process. Technol. 75 (1998) 222-234.

[10] M. Traversin, R. Kergen, Closed-loop control of the blank-holder force in deep-drawing: finite-element modeling of its effects and advantages, J. Mater. Process. Technol. 50 (1995) 306-317.

[11] L. Gunnarsson, E. Schedin, Improving the properties of exterior body panels in automobiles using variable blank holder force, J. Mater. Process. Technol. 114 (2001) 168-173.

[12] N. Krishnan, J. Cao, Estimation of optimal blank holder force trajectories in segmented binders using an ARMA model, J. Mech. Sci. Eng. 125 (2003) $763-770$.

[13] Z.Q. Sheng, S. Jirathearanat, T. Altan, Adaptive FEM simulation for prediction of variable blank holder force in conical cup drawing, Int. J. Mach. Tools Manuf. 44 (2004) 487-494.

[14] S. Yoshihara, K.-I. Manabe, H. Nishimura, Effect of blank holder force control in deep-drawing process of magnesium alloy sheet, J. Mater. Process. Technol. 170 (2005) 579-585.

[15] D.-K. Leu, The limiting drawing ratio for plastic instability of the cupdrawing process, J. Mater. Process. Technol. 86 (1999) 168-176.

[16] L. Duchêne, A.M. Habraken, Analysis of the sensitivity of FEM predictions to numerical parameters in deep drawing simulations, Eur. J. Mech. A/Solids 24 (2005) 614-629.

[17] R.K. Verma, S. Chandra, An improved model for predicting limiting drawing ratio, J. Mater. Process. Technol. 172 (2006) 218-224.

[18] D.W.A. Rees, Sheet orientation and formability limits under diffuse necking, Appl. Math. Modelling 20 (8) (1996) 624-635.

[19] D. Ravikumar, Formability analysis of extra-deep-drawing steel, J. Mater. Process. Technol. 130-131 (2002) 31-41.

[20] A.G. Mamalis, D.E. Manolakos, A.K. Baldoukas, Simulation of sheet metal forming using explicit finite-element techniques: effect of material and forming characteristics, Part 1. Deep-drawing of cylindrical cups, J. Mater. Process. Technol. 72 (1997) 48-60.

[21] L.F. Menezes, C. Teodosiu, Three-dimensional numerical simulation of the deep-drawing process using solid finite elements, J. Mater. Process. Technol. 97 (1-3) (2000) 100-106.

[22] J.M. Antunes, L.F. Menezes, M.F. Vieira, J.V. Fernandes, B. Trindade, A.S. Ramos, M.T. Vieira, On the evaluation of the ductility of thin films, Mater. Sci. Eng. A 337 (2002) 97-103.

[23] R. Hill, A theory of the yielding and plastic flow of anisotropic metals, Proceeding of the R. Soc. London Ser. A 193 (1948) 281-297.

[24] S.H. Park, Robust Design \& Analysis for Quality Engineering, Chapman \& Hall, London, 1996. 